\title{
Highly Efficient Side-Coupled Acousto-Optic Modulation of a Suspended Core Fiber Bragg Grating
}

\author{
Ricardo E. da Silva, Egor Manuylovich, Namita Sahoo, Martin Becker, Manfred Rothhardt, \\ Hartmut Bartelt and David J. Webb
}

\begin{abstract}
We demonstrate acousto-optic modulation of a fiber Bragg grating in a birefringent suspended core fiber up to frequencies of $5.2 \mathrm{MHz}$ for the first time. At acoustic resonances lower than $820 \mathrm{kHz}$, the reflectivity of the orthogonal polarization modes is electrically switched to a superposed reflection band with a maximum modulation depth of $51 \%$ at $10 \mathrm{~V}$. The wavelength peak of the polarization modes is dynamically shifted by tuning the driven electrical signal from 1.92 to 5.2 MHz. A novel side-coupled based acousto-optic device is demonstrated, indicating new possibilities for ultracompact, fast and efficient all-fiber integrated devices.
\end{abstract}

Index Terms - acousto-optic devices, fiber Bragg gratings, fiber-optic components.

\section{INTRODUCTION}

$\mathrm{O}$ PTICAL devices based on fiber Bragg gratings (FBGs) and long period gratings (LPGs) have attracted significant interest because of their potential application in spectral filters, fiber sensors and fiber lasers [1]-[4]. In particular, FBGs combined with birefringent fibers enable unique possibilities to generate and tune multiwavelength fiber lasers [3], [4]. The generation of spectral channels with tunable reflectivity or wavelength is usually achieved by changing the properties of the orthogonal polarization modes by means of strain, curvature, or temperature methods. Nevertheless, most of the proposed techniques are relatively slow to change the optical properties, consequently limiting the fast tuning of the devices. Electrical tuning of the effective refractive indices of the polarization modes employing high voltage thermal based techniques $(160-190 \mathrm{~V})$ has been successfully demonstrated [5]. However, the device response time is usually limited to the tens of milliseconds range of $35-70 \mathrm{~ms}$ because of the slow material heating/cooling process. Overall, the combination of polarization controllers, interferometers or additional components in the setup may induce high insertion losses, increasing the device complexity, size and costs.

Alternatively, acousto-optic devices offer fast tuning of the power and spectral properties of optical fibers by electricallytunable acoustic waves with response times in the range of $42-$

This project has received funding from the European Union's Horizon 2020 research and innovation programme under the Marie Sklodowska-Curie grant agreement No 713694. Ricardo E. da Silva (e-mail: r.da-silva@aston.ac.uk ), Egor Manuylovich, Namita Sahoo and David J. Webb are with the Aston Institute of Photonic Technologies (AIPT), Aston University, Birmingham, B4 7ET, UK. Martin Becker, Manfred Rothhardt and Hartmut Bartelt are with the
$150 \mu \mathrm{s}$ [6]-[8]. Nevertheless, the commonly used standard single-mode fibers (SMFs) provide a poor overlap between the acoustic waves and the optical modes or gratings in the fiber core, since the acoustic power is distributed over the whole fiber cross section. The modulators generally require long interaction lengths to enhance the acousto-optic efficiency (device lengths longer than $6.9 \mathrm{~cm}$ have been reported [7]-[10]). The use of long fiber lengths however increases the device response time because the acoustic waves take more time to travel along the fiber. Cladding-etched fibers or tapered fibers strengthen the acousto-optic interaction [7], [8], [11]. However, the excessive reduction of the fiber diameter might cause mechanical instabilities, exposing the optical properties to surface impurities. In this sense, suspended-core fibers (SCFs) have emerged as a promising alternative to enhance the interaction of acoustic and optical power in the fiber core without reducing the cladding diameter [12], [13].

In this Letter, we demonstrate new efficient and fast sidecoupled acoustically induced modulation of a FBG in a highly birefringent suspended core fiber (HB-SCF) up to $5.2 \mathrm{MHz}$. The HB-SCF supports two polarization modes propagating in the core with distinct effective indices $n_{\text {eff }}$ and phase velocities $v$, usually named as "fast $\mathrm{Y}$ " and "slow X" modes. Interaction of these modes with a grating results in two reflected bands centered at the wavelengths $\lambda_{\mathrm{Y}}$ and $\lambda_{\mathrm{X}}$ (unmodulated FBG in Fig. 1(a)). The grating supports degenerated polarization modes $\mathrm{XYC}$, propagating in phase and overlapping the grating reflectivity leading to a superposed central band centered at $\lambda_{\mathrm{C}}$, as illustrated in Fig. 1(a) [12].

The acoustically induced strain modulates the grating period $\Lambda(z)$ and effective refractive index $n_{\text {eff }}$ of the fast and slow modes, inducing reflection lobes on both sides of the polarization peaks. The modulated power from the fast mode at the inner right sidelobe $\lambda_{\mathrm{YC}}$, propagates in a longer optical path length and grating period (tensioned grating) compared to the slow mode at the left sidelobe $\lambda$ xc (compressed grating), compensating the velocities of the orthogonal modes to match in phase with the degenerated central mode at $\lambda_{\mathrm{C}}[12]$. Thus, the inner lobes are tuned by the acoustic frequency to superimpose

Leibniz Institute of Photonic Technology (IPHT), Albert-Einstein-Straße 9, 07745 Jena, Germany. Copyright (c) 2021 IEEE. Personal use of this material is permitted. However, permission to use this material for any other purposes must be obtained from the IEEE by sending a request to pubspermissions@ieee.org. 
(a)

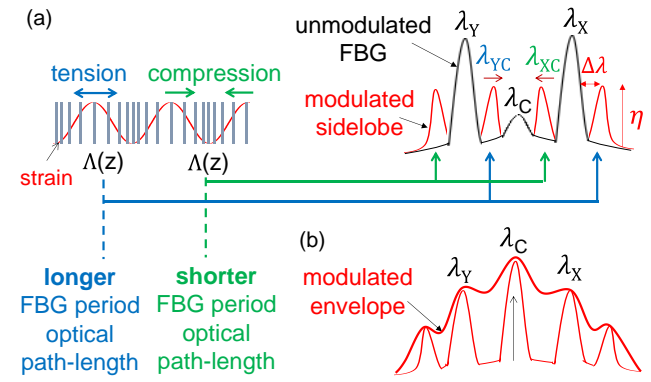

Fig. 1. Illustration of the (a) unmodulated and modulated FBG spectrum in the HB-SCF by means of a longitudinal acoustic wave. (b) The modulated inner sidelobes overlap in phase inducing a superposed reflection band at $\lambda_{\mathrm{c}}$.

the reflectivities at $\lambda_{\mathrm{C}}$, resulting in a multiwavelength modulated envelope (Fig. 1(b)).

The sidelobe reflectivity $\eta$ and separation $\Delta \lambda$ are [11],

$$
\begin{gathered}
\eta=\tanh ^{2}\left[\frac{\pi \Delta n_{a c} \Gamma}{\lambda_{B}} L_{g} J_{m}\left(\frac{\lambda_{a}}{\Lambda} \sqrt{\frac{2 P_{a c}}{Y A_{s} v_{e x t}}}\right)\right], \\
\Delta \lambda=\frac{f \lambda_{B}^{2}}{2 n_{\text {eff }} v_{\text {ext }}},
\end{gathered}
$$

in which, $\lambda_{B}=2 n_{\text {eff }} \Lambda$ is the Bragg wavelength, $J_{m}$ is the Bessel function of the first kind of order $m, \Delta n_{a c}$ is the grating index modulation amplitude, $L_{g}$ is the grating length, $\lambda_{a}$ is the acoustic period, $\Gamma$ is the confinement factor, $P_{a c}$ is the acoustic power, $Y$ is the Young's modulus, $A_{s}$ is the silica fiber cross section and, $v_{\text {ext }}$ is the extensional acoustic velocity in silica. The reflectivity of the polarization peaks and superposed central band is accordingly tuned by adjusting the sidelobe reflectivity $\eta$ and separation $\Delta \lambda$ with the acoustic power $P_{a c}$ and frequency $f$, respectively.

\section{EXPERIMENTAL SETUP}

Fig. 2(a) illustrates the acousto-optic device and the setup used to characterize the modulated FBG spectrum. The modulator is fabricated with a piezoelectric transducer (PZT), an acoustic horn and the HB-SCF. The fiber is composed of two similar pure silica cores $(7.7 \mu \mathrm{m} \times 4 \mu \mathrm{m})$ and four air holes (diameter range $40-43 \mu \mathrm{m}$ ) separated by silica bridges of $2.7 \mu \mathrm{m}$ thickness. Fig. 2(b) shows the HB-SCF cross section with a detail of one of the fiber cores in Fig. 2(c). The fiber diameter is $124 \mu \mathrm{m}$. A $1 \mathrm{~cm}$ long FBG is inscribed in the fiber by means of a femtosecond laser, using a phase mask interferometer and two-beam interference technique, according to the method described in [14].

The PZT disc is $200 \mu \mathrm{m}$ thickness and $3 \mathrm{~mm}$ in diameter. The acoustic horn is made by tapering a solid silica rod from 640 to $80 \mu \mathrm{m}$ in diameter along $1.6 \mathrm{~cm} \mathrm{[10].} \mathrm{The} \mathrm{horn} \mathrm{is} \mathrm{connected} \mathrm{to}$ the PZT and fiber by means of a glass adhesive. The horn tip is fixed on the fiber surface along $3 \mathrm{~mm}$, forming the sidecoupling connection, as illustrated in Fig. 2(a). The PZT base is fixed on a metallic support connecting to a signal generator (SG). Conductive glue electrically connects the metallic support and PZT, preventing the use of conventional soldering causing unsuitable loads on the PZT. The modulator works as a resonant acoustic cavity at discrete resonance frequencies by fixing the PZT base and the fiber. The HB-SCF is spliced to the

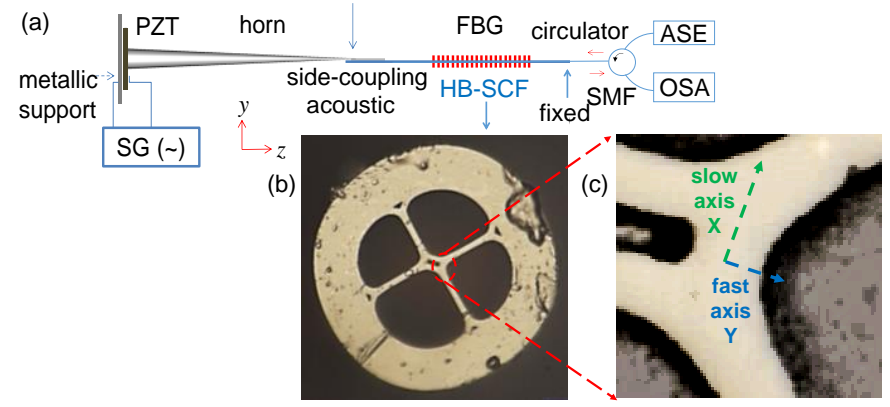

Fig. 2. (a) Illustration of the acousto-optic modulator and experimental setup, with details of the (b) HB-SCF cross section and (c) one of the fiber cores.

SMF connecting to a circulator using an arc-discharge fusion splicer. The whole modulator is $3.2 \mathrm{~cm}$ long.

The PZT is excited by a sinusoidal electrical signal from 1 to $10 \mathrm{~V}$ (peak-to-peak) at the resonances of $f=215 \mathrm{kHz}$, $f=353 \mathrm{kHz}$ and $f=820 \mathrm{kHz}$. The modulator is further tuned at the resonances of $f=1.95 \mathrm{MHz}, f=2.92 \mathrm{MHz}$ and $f=5.2 \mathrm{MHz}$ with a constant $10 \mathrm{~V}$. The best performance is achieved at the resonance frequencies, which depend on the component's material and dimensions and overall device size. The acoustic waves are amplified by the acoustic horn and coupled by the fiber surface to interact with the grating. The FBG spectrum is characterized by employing a broadband optical source (ASE730 Thorlabs) and an optical spectrum analyzer (OSA Yokogawa AQ6370D) with a $20 \mathrm{pm}$ wavelength resolution. The temporal response of the modulator is characterized by tuning a $\mathrm{CW}$ laser at the modulated superposed peak $\lambda_{\mathrm{C}}$ and the resonance of $f=820 \mathrm{kHz}$. The modulated optical power is measured with a photodetector and an oscilloscope.

\section{RESULTS AND DiscuSSIONS}

Fig. 3 shows the unmodulated FBG spectrum indicating the peaks of the orthogonal polarization modes centered at $\lambda_{\mathrm{Y}}=1540.32 \mathrm{~nm}$ and $\lambda_{\mathrm{X}}=1540.58 \mathrm{~nm}$ (voltage off). The spectra are normalized to the maximum reflectivity. The centered wavelength of the superposed XY mode is estimated at $\lambda_{\mathrm{C}}=1540.46 \mathrm{~nm}$, being $130 \mathrm{pm}$ (averaged value) separated from the polarization peaks. The modulated grating reflectivity is evaluated at these fixed peak values for the voltage range of $1-10 \mathrm{~V}$ applied to the PZT at the resonances of $f=215 \mathrm{kHz}$, $f=353 \mathrm{kHz}$ and $f=820 \mathrm{kHz}$, as indicated in Fig. 3. The respective sidelobe separation, $\Delta \lambda=31 \mathrm{pm}, \Delta \lambda=51 \mathrm{pm}$ and $\Delta \lambda=118 \mathrm{pm}$, are calculated with Eq. (2), considering the averaged modal effective index $n_{\text {eff }}=1.43$ (estimated from the polarization peaks) and an extensional silica velocity of $v_{\text {ext }}=5740 \mathrm{~m} / \mathrm{s}$ [9]). Analysis indicates that the modulated peak at $\lambda_{\mathrm{C}}$ in Figs. 3(a) and 3(b) is also induced by the overlapping of higher order lobes with the separation being a multiple of the first-order sidelobes in Eq. (2) (e.g., $2 \Delta \lambda, 3 \Delta \lambda$, etc.) [11].

Fig. 3(a) shows four sidelobe peaks over the modulated envelope at 7 and $8 \mathrm{~V}$ (indicated with green circles at $7 \mathrm{~V}$ ). The increasing voltage modulates the superposed central band with a maximum depth of $\Delta \eta_{\mathrm{C}}=41 \%$ at $f=215 \mathrm{kHz}$. This increased reflectivity is followed by a decreasing reflection of the fast and slow peaks, achieving a modulation of $\Delta \eta_{\mathrm{Y}}=51 \%$ and $\Delta \eta_{\mathrm{X}}=47 \%$, respectively. For the resonance of $f=353 \mathrm{kHz}$ in 

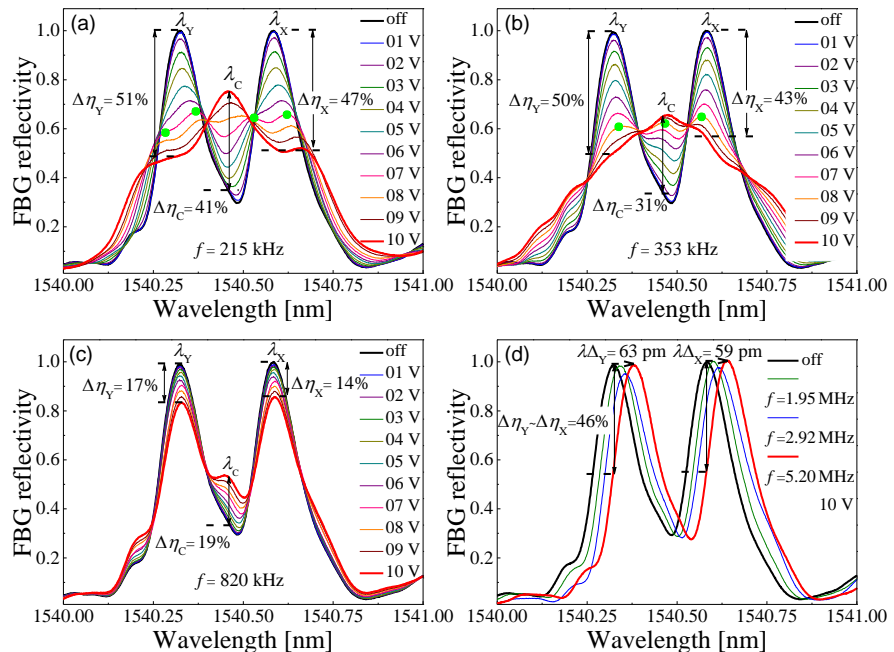

Fig. 3. Unmodulated (voltage off) and modulated FBG spectra for the resonances at (a) $f=215 \mathrm{kHz}$, (b) $f=353 \mathrm{kHz}$ and (c) $f=820 \mathrm{kHz}$, and the voltage range of 1-10 V applied to the PZT. (d) Unmodulated (voltage off) and modulated FBG spectra for the resonances at $f=1.95 \mathrm{MHz}, f=2.92 \mathrm{MHz}$ and $f=5.2 \mathrm{MHz}$, and the voltage of $10 \mathrm{~V}$ applied to the PZT.

Fig. 3(b), the peaks' modulation slightly decreases to $\Delta \eta_{\mathrm{Y}}=50 \%$ and $\Delta \eta_{\mathrm{X}}=43 \%$. The values are measured considering the modulated envelope as reference. In fact, values higher than $\Delta \eta=60 \%$ are observed at the outside edges of the polarization peaks. Note three sidelobe peaks over the modulated envelope at 7 and $8 \mathrm{~V}$. It is caused by a larger sidelobe separation compared to the resonance in Fig. 3(a). Nevertheless, the superposed band at $\lambda_{\mathrm{C}}$ decays to $\Delta \eta_{\mathrm{C}}=31 \%$ indicating detuning between the sidelobes and the coupling wavelength $\lambda_{\mathrm{C}}$. The peaks' visibility is improved by measuring the modulated FBG spectra with an OSA or other equipment with higher wavelength resolution. The polarization properties of this kind of gratings are studied in [12]. The modulation of higher order lobes is significantly reduced at $f=820 \mathrm{kHz}$, as seen in Fig. 3(c). Consequently, switching from the orthogonal modes directly to the superposed mode is more evident for this resonance. However, the reduced acoustic amplitude and detuning contribute to lower modulation values of the polarization peaks $\left(\Delta \eta_{\mathrm{Y}}=17 \%\right.$ and $\left.\Delta \eta_{\mathrm{X}}=14 \%\right)$ and the superposed central band $\left(\Delta \eta_{\mathrm{C}}=19 \%\right)$.

The modulation depth $\Delta \eta$ variation at the peaks $\lambda_{\mathrm{Y}}, \lambda_{\mathrm{X}}$ and $\lambda_{\mathrm{C}}$ with increasing voltage is summarized for the considered resonances in Fig. 4(a). Note that the modulated reflection of the fast mode, $\Delta \eta_{\mathrm{Y}}$, is slightly higher than the slow mode, $\Delta \eta_{\mathrm{X}}$. This difference might be caused by the peak's asymmetry (the spectrum of the fast peak is slightly broader than the slow peak, indicating a higher confinement factor in Eq. (1)). It suggests higher spectral power confined in the fast peak band overlapping with the grating in the fiber core, consequently inducing higher modulation. The modulated reflectivity $\eta$ also depends on the effective index $n_{\text {eff, }}$ considering $\lambda_{B}=2 n_{\text {eff }} \Lambda$ in Eq. 1. Thus, the smaller effective index of $\lambda_{Y}$ contributes to higher $\eta$. Overall, considering that the sidelobes are replicas of the Bragg reflection band [11], asymmetries in the unmodulated peaks are also expected in the modulated spectrum. Nonuniform distribution of the acoustic strain over the fiber cross section caused by the side-coupling is also expected [1]. The narrowest core region in Fig. 2(c) might benefit to concentrate the strain in the core center, inducing increased modulation of the fast mode. Overall, the longitudinal acoustic waves are efficiently coupled by means of the fiber surface. The acoustic period of the respective resonances, $\lambda_{a-215 \mathrm{kHz}}=2.7 \mathrm{~cm}, \lambda_{a-}$ $353 \mathrm{kHz}=1.6 \mathrm{~cm}$, and $\lambda_{a-820 \mathrm{kHz}}=0.7 \mathrm{~cm}\left(v_{\text {ext }}=f \lambda_{a}\right)$ are much longer compared to the $3 \mathrm{~mm}$ long side-coupling connection, favoring the axially induced strains along the grating.

A strong modulated wavelength shift of both polarization modes is revealed for the resonances of $f=1.95 \mathrm{MHz}$, $f=2.92 \mathrm{MHz}$ and $f=5.2 \mathrm{MHz}$, as shown in Fig. 3(d). The fast and slow peaks shift by a maximum of $\lambda \Delta_{\mathrm{Y}}=63 \mathrm{pm}$ and $\lambda \Delta_{\mathrm{X}}=59$ pm, respectively. A modulation depth of $\Delta \eta=46 \%$ is consequently induced at both polarization peaks. The peaks' induced shift is almost linear with increasing frequency, as shown in Fig. 4(b). The estimated acoustic wavelengths at these resonances are $\lambda_{a-1.95 \mathrm{MHz}}=2.9 \mathrm{~mm}, \lambda_{a-2.92 \mathrm{MHz}}=2 \mathrm{~mm}$ and $\lambda_{a-}$ $5.20 \mathrm{MHz}=1.1 \mathrm{~mm}$, respectively. The decreasing acoustic period to values smaller than the $3 \mathrm{~mm}$ horn-fiber connection implies a non-uniform distribution of the acoustic wave along the fiber. In this case, the central air hole and the fiber cores shifted in relation to the fiber center in Fig. 2(b), likely contribute to an asymmetric distribution of the induced tensive and compressive strains over the fiber cores and silica bridges.

At lower frequencies, the symmetric longitudinal acoustic waves induce tension and compression strains along the grating, inducing respective right and left lobes besides the $\lambda_{\mathrm{Y}}$ and $\lambda_{\mathrm{X}}$ peaks in Fig. 1(a). Both strains are uniformly distributed over the SCF cross section with almost equivalent lengths along the grating. The lobes are considered as a partial dynamic shift of the peaks simultaneously to both sides. Consequently, the inner lobes generate the superposed central band. At higher frequencies, the side-coupled asymmetric acoustic waves induce distinct tensive and compressive strains in the out-ofcenter fiber cores over the SCF cross section. As a result, tension predominates along the grating in the evaluated fiber core, shifting the polarization peaks only to the right (Fig. 3(d)).

We have investigated the best time response of the acoustooptic modulator at $f=820 \mathrm{kHz}$. Fig. 4(c) shows the electrical signal applied to the PZT and the modulated optical signal at $\lambda_{\mathrm{C}}$ and $f=820 \mathrm{kHz}$. The acoustic wave takes $\tau_{0-100 \%}=12.4 \mu$ s to raise the optical signal from the lower to the upper stationary level $(0-100 \%)$. The delay between the electrical and optical signals of $\tau_{\mathrm{DL}}=3.5 \mu \mathrm{s}$ is related to the acoustic wave generation and propagation along the horn (it is up to 14 times shorter compared to previous reports of $25-50 \mu$ s [6][7]. The overall device switching time is about $\tau_{\mathrm{ST}}=16 \mu \mathrm{s}$. Comparable modulation values of $\Delta \eta_{\mathrm{Y}}=50 \%$ are efficiently achieved by the side-coupling employing a significant smaller PZT (10 times in thickness and 8 times in diameter), smaller acoustic horn (8 times base diameter and 3 times in length), and an overall smaller modulator (the $3.2 \mathrm{~cm}$ long device is about 3 times smaller compared to previous similar devices of about $9.5 \mathrm{~cm}$ [6][12]). The side-coupling might enable operation in transmission, employing simpler solid acoustic horns compared to the hollow horns made with complex fabrication techniques [10]. The PZT and horn could be slightly shifted from the fiber axis providing access to both fiber ends (the small horn diameter of $80 \mu \mathrm{m}$ offers bending flexibility of this purpose). 


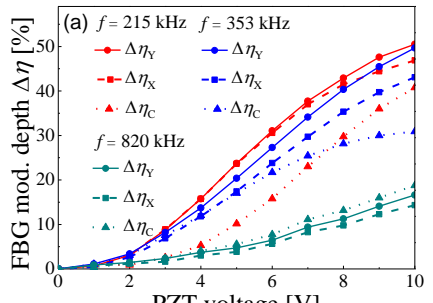

PZT voltage [V]

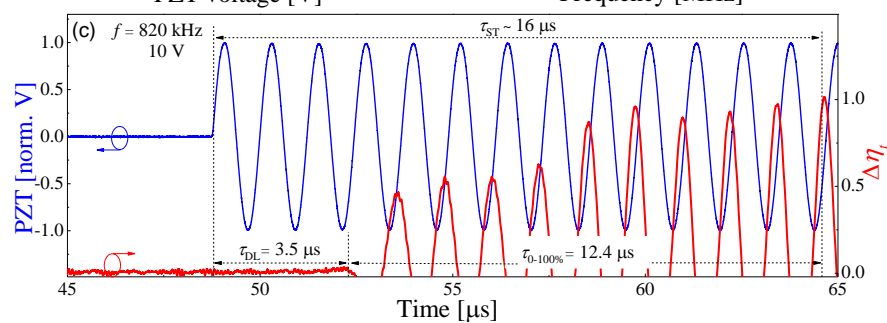

Fig. 4. (a) FBG modulation at the polarization peaks and superposed band with increasing voltage at the resonances of $f=215 \mathrm{kHz}, f=353 \mathrm{kHz}$ and $f=820 \mathrm{kHz}$. (b) Modulated depth and wavelength of the polarization peaks with increasing frequency at the resonances of $f=1.95 \mathrm{MHz}, f=2.92 \mathrm{MHz}$ and $f=5.2 \mathrm{MHz}$. (c) Time response of the acousto-optic modulator.

The modulation depth $\Delta \eta$ depends on the modulator design, efficiency of the PZT, acoustic horn and fiber, coupling between the components and with the electrical circuit (e.g. adhesives, electrodes and soldering connections). The suspended core fibers are the main contributors because of the considerable reduction of the effective silica cross section area, $A_{s}$, in Eq. (1). Thus, the increased HB-SCF efficiency is also predicted for other kinds of SCFs [13], being promising for highly efficient modulation with increasing frequency [15].

The modulation depth $\Delta \eta$ might be increased by improving the components' efficiency and connections (e.g. highly efficient PZTs with reduced diameters matching with the cross section of shorter silica horns). Improved efficiency might also be achieved by employing FBGs with high grating index modulation amplitudes $\Delta n_{a c}$, inscribed in SCFs with larger air holes. Long grating lengths $L_{g}$ (e.g. $L_{g}=5 \mathrm{~cm}$ ) enhance the modulator efficiency, as indicated in Eq. (1) [7]. However, long $L_{g}$ increases the device size and the response time. As demonstrated, the reduced $3.2 \mathrm{~cm}$ modulator works with a short switching time $\left(\tau_{\mathrm{ST}}=16 \mu \mathrm{s}\right)$. Given the size of existing modulators, such as, electro-optic devices based on 2Dmaterials [16], it is desirable to reduce the size of our device still further. The acousto-optic modulator might be reduced to a millimeter scale by confining the side-coupled acoustic waves inside the suspended core fiber [15]. Overall, the best grating length is defined by a compromise between the modulation efficiency, response time and device size required for a specific application.

Higher modulation depth and shorter response times are also expected by splicing the horn tip and the HB-SCF along the side-coupling connection. Accordingly, a longer wavelength shift range of the peaks at resonances from $f=1.95 \mathrm{MHz}$ is also expected. For frequencies higher than $f=5 \mathrm{MHz}$, the tension and compression regions along the grating $(552 \mu \mathrm{m}$ in length at $f=5.2 \mathrm{MHz}$ ) tend to decrease to the order of the fiber diameter. It will potentially enable confinement of acoustic waves inside suspended core fibers, strengthening the interaction with optical modes and gratings in the fiber core [15].

\section{CONCLUSION}

In summary, we demonstrate highly efficient side-coupled acoustically induced FBG modulation of the polarization modes in a suspended core fiber up to $5.2 \mathrm{MHz}$. A maximum modulation depth of $51 \%$ is achieved at $215 \mathrm{kHz}$ and $10 \mathrm{~V}$. Up to 5 wavelength bands are tuned by the voltage and the frequency of an electrical signal. The polarization peaks shift to longer wavelengths with a maximum of $63 \mathrm{pm}$ at $f=5.2 \mathrm{MHz}$. For the best of our knowlegde, the $3.2 \mathrm{~cm}$ long modulator is the smallest all-fiber acousto device reported, pointing to a new generation of ultracompact and fast fiber-integrated devices.

\section{REFERENCES}

[1] C. Jiang, Y. Liu, and C. Mou, "Polarization-maintaining fiber long-period grating based vector curvature sensor," IEEE Photonics Technol. Lett., vol. 33, no. 7, pp. 358-361, Apr. 2021.

[2] K. Liu et al., "Broadband vibration sensor using reflected excessively tilted fiber grating with clamped beam," IEEE Photonics Technol. Lett., vol. 33, no. 8, pp. 379-382, Apr. 2021.

[3] L. Zhang et al., "Switchable multi-wavelength thulium-doped fiber laser employing a polarization-maintaining sampled fiber bragg grating," IEEE Access, vol. 7, pp. 155437-155445, 2019.

[4] M. Zou et al., "Multiwavelength mode-locked fiber laser based on an all fiber Lyot filter," IEEE Photonics Technol. Lett., vol. 32, no. 22, pp. 1419 1422, Nov. 2020.

[5] P. Xue et al., "Electrically tuning characteristics of LC selectively infiltrated pcf sagnac interferometer," IEEE Photonics Technol. Lett., 2021.

[6] C. A. F. Marques, R. A. Oliveira, A. A. P. Pohl, J. Canning, and R. N. Nogueira, "Dynamic control of a phase-shifted FBG through acousto-optic modulation," Opt. Commun., vol. 284, no. 5, pp. 1228-1231, Mar. 2011.

[7] M. Delgado-Pinar, D. Zalvidea, A. Diez, P. Perez-Millan, and M. Andres, "Q-switching of an all-fiber laser by acousto-optic modulation of a fiber Bragg grating," Opt. Express, vol. 14, no. 3, pp. 1106-12, Feb. 2006.

[8] J. Lu et al., "Recent progress of dynamic mode manipulation via acoustooptic interactions in few-mode fiber lasers: Mechanism, device and applications," Nanophotonics, vol. 10, no. 3, pp. 983-1010, Jan. 2021.

[9] R. E. Silva, M. Becker, M. Rothhardt, H. Bartelt, and A. A. P. Pohl, "Acousto-optic notch filter dynamically induced in a chirped fiber Bragg grating," IEEE Photonics Lett., vol. 28, no. 10, pp. 1081-1083, May 2015.

[10] R. E. Silva et al., "All-fiber $10 \mathrm{MHz}$ acousto-optic modulator of a fiber Bragg grating at $1060 \mathrm{~nm}$ wavelength," Opt. Express, vol. 23, no. 20, pp. 25972-8, Oct. 2015.

[11] W. F. Liu, P. S. J. Russell, and L. Dong, "100\% efficient narrow-band acoustooptic tunable reflector using fiber Bragg grating," J. Light. Technol., vol. 16, no. 11, pp. 2006-2009, 1998.

[12] R. E. Silva, M. Becker, M. Rothhardt, H. Bartelt, and A. A. P. Pohl, "Electrically tunable multiwavelength Bragg grating filter acoustically induced in a highly birefringent suspended core fiber," IEEE Photonics J., vol. 9, no. 1, pp. 1-9, Feb. 2017.

[13] R. E. Silva, M. Becker, M. Rothhardt, H. Bartelt, and A. A. P. Pohl, "Acousto-optic double side-band amplitude modulation of a fiber Bragg grating in a four-holes suspended-core fiber," J. Light. Technol., vol. 36, no. 18, pp. 4146-4152, Sep. 2018.

[14] M. Becker et al., "Fiber Bragg grating inscription combining DUV subpicosecond laser pulses and two-beam interferometry," Opt. Express, vol. 16, no. 23, p. 19169, Nov. 2008.

[15] R. E. Silva and D. J. Webb, "Ultra-efficient in-core acoustic waves in suspended core fiber for high frequency fiber-optic ultrasonic devices," Appl. Phys. Express, vol. 14, no. 8, p. 087003, Aug. 2021.

[16] J. Chen, Y. Xiong, F. Xu, and Y. Lu, "Silica optical fiber integrated with two-dimensional materials: towards opto-electro-mechanical technology," Light Sci. Appl. 2021 101, vol. 10, no. 1, pp. 1-18, Apr. 2021. 